\title{
On stability analysis of discrete-time uncertain switched nonlinear time-delay systems
}

\author{
Marwen Kermani* and Anis Sakly
}

"Correspondence:

kermanimarwen@gmail.com Research Unit of Industrial Systems

Study and Renewable Energy (ESIER), Department of Electrical Engineering, National Engineering School of Monastir (ENIM), Ibn El Jazzar, Skaness, Monastir, 5019, Tunisia

\begin{abstract}
This paper addresses the stability analysis problem for a class of discrete-time switched nonlinear time-delay systems with polytopic uncertainties. These considered systems are characterized by delayed difference nonlinear equations which are given in the state form representation. Then, a transformation under the arrow form is employed. Indeed, by constructing an appropriated common Lyapunov function, and also by resorting to the Kotelyanski lemma and the $M$-matrix proprieties, new delay-independent stability conditions under arbitrary switching law are deduced. Compared with the existing results of switched systems, those obtained results are formulated in terms of the unknown polytopic uncertain parameters, explicit and easy to apply. Moreover, this method allows us to avoid the search for a common Lyapunov function which is a difficult matter. Finally, a numerical example is presented to illustrate the effectiveness of the proposed approach.
\end{abstract}

Keywords: discrete-time switched nonlinear time-delay systems; polytopic uncertainties; global robust asymptotic stability; M-matrix properties; Kotelyanski lemma; arrow matrix; arbitrary switching

\section{Introduction}

Switched systems are an important class of hybrid systems. Generally speaking, a switched system is composed of a family of subsystems described by differential or difference equations and a switching rule orchestrating the switching between the subsystems that have attracted much attention in control theory and practice during recent decades. Switched systems can be efficiently used to model many practical systems which are inherently multi-model in the sense that several dynamical systems are required to describe their behavior. For example, many physical processes exhibit switched and hybrid nature. Switched systems have strong engineering background in various areas and are often used as a unified modeling tool for a great number of real-world systems, such as power electronics, chemical processes, mechanical systems, automotive industry, aircraft and air traffic control and many other fields [1-6].

Undoubtedly, stability is the first requirement for a system to work properly; thus, stability analysis of switched systems presents a theoretical challenge, which has attracted growing attention in the literature [1-37]. However, stability under arbitrary switching is a fundamental issue and an important topic in the design and analysis of these systems.

○2014 Kermani and Sakly; licensee Springer. This is an Open Access article distributed under the terms of the Creative Commons Attribution License (http://creativecommons.org/licenses/by/2.0), which permits unrestricted use, distribution, and reproduction in any medium, provided the original work is properly cited. 
To solve this matter, many effective methods have been developed [11, 15, 16, 24]. Within this framework, we are required to find conditions that guarantee asymptotic stability under arbitrary switching rules. Indeed, it is well known that the existence of a common Lyapunov function [7-9] for all subsystems is sufficient to guarantee the stability under arbitrary switching law. However, finding such a function is often difficult even for discretetime switched linear systems [37]. Consequently, this problem becomes more complicated for discrete-time switched nonlinear systems, and relatively fewer results have been reported in this context.

On the other hand, to avoid the problem of the existence of a common Lyapunov function, some attention has been widely given to seeking conditions that guarantee the stability of the switched systems under restricted switching. Although many efficient approaches and important results have been proposed for this alternative, such as the multiple Lyapunov function approach [10] and average dwell time method [11, 12], stability under arbitrary switching, which is considered in this work, remains most preferable for practical systems. Indeed, it offers great flexibility and it allows us to achieve other performances for designing a control law along stability maintained.

As is well known, time-delay phenomena are usually confronted in many engineering systems $[1,16-24,28-30,38,39]$, such as chemical engineering systems, hydraulic systems, inferred grinding model, neural network, nuclear reactor, population dynamic model and rolling mill. Recently, stability analysis for discrete-time switched time-delay systems has been investigated $[1,16-24,28-30]$.

It is noteworthy that there are two divisions in the recent literature addressing the stability analysis of time-delay systems, namely delay-independent criteria and delay-dependent criteria. Therefore, in view of delay-independent criteria, this paper will try to aid the stability analysis under arbitrary switching law.

On the other hand, when practical systems are modelled, uncertainties of system parameters are often included. Therefore, most of the systems refer to uncertainties in real applications. Indeed, basically, two kinds of uncertainties are simultaneously encountered in the open literature, widely polytopic uncertainties and norm-bounded. From the practical viewpoint, it is important to investigate switched systems with uncertain parameters $[19,20,24,28-30,33,36]$. Thus, polytopic uncertainties exist in many real systems, and most of the uncertain systems can be approximated by systems with polytopic uncertainties [34]. On the other hand, the polytopic uncertain systems are less conservative than systems with norm-bounded uncertainties [35].

Up to now, discrete-time uncertain switched time-delay systems have received more and more attention. Although many interested and significant results on stability problems for those systems have been established [19, 20, 26, 28-30], those previous works were mainly focused on several hot topics of Lyapunov stability theory and most of them were interested in the linear case $[19,22-26,28-30]$. Thus, due to the complexity of switched nonlinear systems, unfortunately, the available results on the stability of uncertain discretetime switched nonlinear time-delay systems are limited [20].

Motivated by these mentioned shortcomings for the existing results as well as in the sense of various methods that can be employed, in this paper we aim to establish new stability analysis for a class of discrete-time switched time-delay systems with polytopic uncertainties. Indeed, based on transforming, the representation of these systems are studied under consideration into the arrow form matrix [31-33, 40-51]. Then, by constructing an 
appropriated common Lyapunov function and by resorting to the Kotelyanski lemma [52] and the $M$-matrix proprieties $[53,54]$, new delay-independent stability conditions under arbitrary switching law are deduced.

Within the frame of studying the stability analysis, the said approach has already been introduced in $[40,41]$ for continuous-time delay systems and in our previous work [31] for discrete-time switched linear time-delay systems in a field related to the study of convergence.

To the best of the authors' knowledge, no results have been reported in the literature on the stability of discrete-time switched time-delay systems with polytopic uncertainties by employing the Kotelyanski stability conditions that can be very effective in dealing with a class of more general nonlinear systems.

The contributions in our work mainly include two aspects. First, due to the conservatism of the methods for stability analysis which are based on the common Lyapunov function, this proposed method can guarantee stability under arbitrary switching and allows us to avoid searching for a common Lyapunov function. Second, these obtained results are formulated in terms of the unknown polytopic uncertain parameters, explicit and easy to apply. Furthermore, this proposed approach could be further used as a constructive solution to the problems of state and static output feedback stabilization.

The layout of the paper is as follows. Section 2 presents the problem formulation and some preliminaries. The main results of this paper are presented in Section 3. Section 4 is devoted to deriving new delay-independent conditions for asymptotic stability of switched nonlinear systems defined by difference equations. Some remarks and a numerical example are presented in Section 5 to illustrate the theoretical results. Finally, Section 6 concludes this paper.

Notation The notation used throughout this paper is as follows. For a matrix $A$, we denote the transpose by $A^{T}$. Let $\Re$ denote the field of real numbers, $\Re^{n}$ denote an $n$-dimensional linear vector space over the reals with the norm $\|\cdot\|$. For any $u=\left(u_{i}\right)_{1 \leq i \leq n}, v=\left(v_{i}\right)_{1 \leq i \leq n} \in$ $\Re^{n}$, we define the scalar product of the vectors $u$ and $v$ as $\langle u, v\rangle=\sum_{i=1}^{n} u_{i} v_{i}$; $\Re^{n \times n}$ is the space of $n \times n$ matrices with real entries. $\Re_{+}$is a set of positive real numbers. $I\left[k_{1} k_{2}\right]$ is a set of integers $\left\{k_{1}, k_{1}+1, k_{1}+2, \ldots, k_{2}\right\}$ and $I_{n}$ is an identity matrix with appropriate dimension.

\section{Problem formulation and preliminaries}

\subsection{Problem formulation}

In this section, consider the discrete-time switched time-delay systems:

$$
\left\{\begin{array}{l}
x(k+1)=A_{\sigma(k)}(\cdot) x(k)+D_{\sigma(k)}(\cdot) x(k-\tau) \\
x(s)=\phi(s), \quad s=-\tau,-\tau+1, \ldots,-1
\end{array}\right.
$$

where $x(k) \in \Re^{n}$ is the state vector of the system at time $k, \tau$ is the time-delay of state, $\phi(s)$ is a vector-valued initial function, $A_{\sigma(k)}(\cdot) \in \Re^{n \times n}, D_{\sigma(k)}(\cdot) \in \mathfrak{R}^{n \times n}$ and $\sigma(k)$ is the switching rule defined by $\sigma(k): \Re_{+} \rightarrow I[1 N]$, assumed to be available in real time. Therefore, the switched system is composed of $N$ discrete-time subsystems which are expressed as 
follows:

$$
x(k+1)=A_{i}(\cdot) x(k)+D_{i}(\cdot) x(k-\tau), \quad i \in I[1 N],
$$

where $A_{i}(\cdot), i \in I[1 N]$ and $D_{i}(\cdot), i \in I[1 N]$ are matrices that have nonlinear elements with appropriate dimensions.

Let $\xi(k)=\left[\xi_{1}(k), \ldots, \xi_{N}(k)\right]^{T}$ be an indication function such that

$$
\xi_{i}(k)= \begin{cases}1 & \text { if } \sigma(k)=i \\ 0 & \text { otherwise }\end{cases}
$$

It is obvious that $\sum_{i=1}^{N} \xi_{i}(k)=1$.

Assume that all subsystems are uncertain systems of polytopic type described as follows:

$$
\begin{aligned}
& A_{i}(\cdot)=\sum_{l=1}^{N_{l}} \mu_{i l}(k) A_{i l}(\cdot), \quad i \in I[1 N], \\
& D_{i}(\cdot)=\sum_{q=1}^{N_{q}} \lambda_{i q}(k) D_{i q}(\cdot), \quad i \in I[1 N],
\end{aligned}
$$

where $A_{i l}(\cdot), l \in I\left[1 N_{l}\right]$ and $D_{i q}(\cdot), q \in I\left[1 N_{q}\right]$ are respectively the vertex matrices denoting the extreme points of the polytope $A_{i}(\cdot), i \in I[1 N]$ and $D_{i}(\cdot), i \in I[1 N], N_{l}$ is the number of the vertex matrices $A_{i}(\cdot), N_{q}$ is the number of the vertex matrices $D_{i}(\cdot)$ and the weighting factors $\mu_{i l}(k), l \in I\left[1 N_{l}\right], \lambda_{i q}(k), q \in I\left[1 N_{q}\right]$ are unknown polytopic uncertain parameters for each $i \in I[1 N]$ belong to $\mu_{i}(k): \sum_{l=1}^{N_{l}} \mu_{i l}(k)=1, \mu_{i l}(k) \geq 0$ and $\lambda_{i q}(k): \sum_{q=1}^{N_{q}} \lambda_{i q}(k)=1$, $\lambda_{i q}(k) \geq 0$.

Then the switched system (2.1) can be written as follows:

$$
\left\{\begin{array}{l}
x(k+1)=\sum_{i=1}^{N} \xi_{i}(k)\left(\sum_{l=1}^{N_{l}} \mu_{i l}(k) A_{i l}(\cdot) x(k)+\sum_{q=1}^{N_{q}} \lambda_{i q}(k) D_{i q}(\cdot) x(k-\tau)\right), \\
x(s)=\phi(s), \quad s=-\tau,-\tau+1, \ldots,-1,0 .
\end{array}\right.
$$

\subsection{Preliminaries}

Now, the following definition, theorem and lemma are preliminarily presented for further development.

For system (2.1) or (2.6) we can give the following definition.

Definition 1 System (2.6) is said to be uniformly robust asymptotically stable if for any $\varepsilon>0$, there is $\delta(\varepsilon)>0$ such that $\max _{-d \leq l \leq 0}\|\phi(k)\|<\delta$ implies $\|x(k, \phi)\| \leq \varepsilon, k \geq 0$. For arbitrary switching law $\sigma(k)$ and all admissible uncertainties (2.4) and (2.5), there is also $\delta^{\prime}>0$ such that $\max _{-d \leq l \leq 0}\|\phi(k)\|<\delta^{\prime}$ implies $\|x(k, \phi)\| \rightarrow 0$ as $k \rightarrow \infty$ for arbitrary switching signal (2.3).

The following lemma and theorem will play an important role in our later development.

Kotelyanski lemma [52] Real parts of the eigenvalues of the matrix $A(\cdot)$, with nonnegative off-diagonal elements, are less than a real number $\mu$ if and only if all those of the matrix $M(\cdot)$, where $M(\cdot)=\mu I_{n}-A(\cdot)$, are positive with $I_{n}$ being the $n$ identity matrix. 
In this case, all the principal minors of matrix $(-A(\cdot))$ are positive. Then, the Kotelyanski lemma permits to deduce the stability properties of the system given by $A(\cdot)$.

Theorem 1 The matrix $A(\cdot)$ is said to be an M-matrix if the following properties are verified:

- All the eigenvalues of $A(\cdot)$ have a positive real part;

- The real eigenvalues are positive;

- The principal minors of $A(\cdot)$ are positive:

$$
(A(\cdot))\left(\begin{array}{cccc}
1 & 2 & \cdots & j \\
1 & 2 & \cdots & j
\end{array}\right)>0, \quad \forall j \in I[1 n]
$$

- For any positive real numbers $\eta=\left(\eta_{1}, \ldots, \eta_{n}\right)^{T}$, the algebraic equations $A(\cdot) x=\eta$ have a positive solution $w=\left(w_{1}, \ldots, w_{n}\right)^{T}$.

Remark 1 A discrete-time system given by a matrix $A(\cdot)$ is stable if the matrix $\left(I_{n}-A(\cdot)\right)$ verifies the Kotelyanski conditions, in this case $\left(I_{n}-A(\cdot)\right)$ is an $M$-matrix.

\section{Main results}

This section will present the main results on the robust stability conditions for the discrete-time nonlinear switched time-delay system with polytopic uncertainties (2.6).

Such sufficient conditions, which are the main theoretical contribution of the paper, are stated in the theorem below.

Theorem 2 System (2.6) is robust asymptotically stable under arbitrary switching rule (2.3) and all admissible uncertainties (2.4) and (2.5) if the matrix $\left(I_{n}-T_{c}(\cdot)\right)$ is an $M$ matrix with

$$
T_{c}(\cdot)=\max _{1 \leq i \leq N}\left(T_{\sigma(k)}(\cdot)\right)
$$

and

$$
T_{\sigma(k)}(\cdot)=\left(\left|A_{\sigma(k)}(\cdot)\right|+\left|D_{\sigma(k)}(\cdot)\right|\right),
$$

where

$$
A_{\sigma(k)}(\cdot)=\left[\begin{array}{cccc}
\sum_{i=1}^{N} \xi_{i}(k) \sum_{l=1}^{N_{l}} \mu_{i l}(k)\left(a_{i l}^{11}(\cdot)\right) & \ldots & \ldots & \sum_{i=1}^{N} \xi_{i}(k) \sum_{l=1}^{N_{l}} \mu_{i l}(k)\left(a_{i l}^{1 n}(\cdot)\right) \\
\vdots & \vdots & \vdots & \vdots \\
\vdots & \vdots & \vdots & \vdots \\
\sum_{i=1}^{N} \xi_{i}(k) \sum_{l=1}^{N_{l}} \mu_{i l}(k)\left(a_{i l}^{n 1}(\cdot)\right) & \ldots & \ldots & \sum_{i=1}^{N} \xi_{i}(k) \sum_{l=1}^{N_{l}} \mu_{i l}(k)\left(a_{i l}^{n n}(\cdot)\right)
\end{array}\right]
$$


and

$$
D_{\sigma(k)}(\cdot)=\left[\begin{array}{cccc}
\sum_{i=1}^{N} \xi_{i}(k) \sum_{q=1}^{N_{q}} \lambda_{i q}(k)\left(d_{i q}^{11}(\cdot)\right) & \ldots & \ldots & \sum_{i=1}^{N} \xi_{i}(k) \sum_{q=1}^{N_{q}} \lambda_{i q}(k)\left(d_{i q}^{1 n}(\cdot)\right) \\
\vdots & \vdots & \vdots & \vdots \\
\vdots & \vdots & \vdots & \vdots \\
\sum_{i=1}^{N} \xi_{i}(k) \sum_{q=1}^{N_{q}} \lambda_{i q}(k)\left(d_{i q}^{n 1}(\cdot)\right) & \ldots & \ldots & \sum_{i=1}^{N} \xi_{i}(k) \sum_{q=1}^{N_{q}} \lambda_{i q}(k)\left(d_{i q}^{n n}(\cdot)\right)
\end{array}\right] .
$$

Proof Let us consider system (2.6) of any switching law (2.3) and all admissible uncertainties (2.4) and (2.5), let $w \in \Re^{n}$ with components $\left(w_{p}>0, \forall p=1, \ldots, n\right)$ and $x(k) \in \mathfrak{R}^{n}$ be the state vector.

Now, define the function below as a common Lyapunov functional

$$
v(x(k), k)=v_{0}(x(k), k)+\sum_{j=1}^{r} v_{j}(x(k), k),
$$

where

$$
\left\{\begin{array}{l}
v_{0}(k)=\langle|x(k)|, w\rangle, \\
v_{j}(k)=\left\langle\left|D_{c}(\cdot)\right||x(k-j)|, w\right\rangle, \quad \forall j=1, \ldots, r
\end{array}\right.
$$

with

$$
D_{c}(\cdot)=\max _{1 \leq i \leq N}\left(D_{\sigma(k)}(\cdot)\right)
$$

Thus, the difference of the Lyapunov functional has the following form:

$$
\begin{aligned}
\Delta v(x(k), k) & =v(x(k+1), k+1)-v(x(k), k) \\
& =\Delta v_{0}(x(k), k)+\sum_{j=1}^{r} \Delta v_{j}(x(k), k) .
\end{aligned}
$$

The above equality can be rewritten as

$$
\begin{aligned}
\Delta v_{0}(x(k), k)+\sum_{j=1}^{r} \Delta v_{j}(x(k), k) \\
=\Delta v_{0}(x(k), k)+\Delta v_{1}(x(k), k) \\
\quad+\Delta v_{2}(x(k), k)+\cdots+\Delta v_{r-1}(x(k), k)+\Delta v_{r}(x(k), k),
\end{aligned}
$$

where

$$
\Delta v_{0}(x(k), k)=\langle|x(k+1)|, w\rangle-\langle|x(k)|, w\rangle
$$

and

$$
\Delta v_{j}(x(k), k)=\left\langle\left|D_{c}(\cdot)\right||x(k-j+1)|, w\right\rangle-\left\langle\left|D_{c}(\cdot)\right||x(k-j)|, w\right\rangle, \quad j=1, \ldots, r .
$$


Using (2.1) we can obtain

$$
\begin{aligned}
\langle|x(k+1)|, w\rangle & =\left\langle\left|A_{\sigma(k)}(\cdot) x(k)+D_{\sigma(k)}(\cdot) x(k-r)\right|, w\right\rangle \\
& <\left\langle\left|A_{\sigma(k)}(\cdot)\right||x(k)|+\left|D_{\sigma(k)}(\cdot)\right||x(k-r)|, w\right\rangle \\
& =\left\langle\left|A_{\sigma(k)}(\cdot)\right||x(k)|, w\right\rangle+\left\langle\left|D_{\sigma(k)}(\cdot)\right||x(k-r)|, w\right\rangle \\
& <\left\langle\left|A_{c}(\cdot)\right||x(k)|, w\right\rangle+\left\langle\left|D_{c}(\cdot)\right||x(k-r)|, w\right\rangle
\end{aligned}
$$

with

$$
A_{c}(\cdot)=\max _{1 \leq i \leq N}\left(A_{\sigma(k)}(\cdot)\right)
$$

By (3.10) and (3.11) we have that

$$
\begin{aligned}
\Delta v(x(k), k)= & \Delta v_{0}(x(k), k)+\sum_{j=1}^{r} \Delta v_{j}(x(k), k) \\
= & \langle|x(k+1)|, w\rangle-\langle|x(k)|, w\rangle+\left(|| D_{c}(\cdot)|| x(k)|, w\rangle-\left\langle\left|D_{c}(\cdot)\right||x(k-1)|, w\right\rangle\right) \\
& +\left(\left\langle\left|D_{c}(\cdot)\right||x(k-1)|, w\right\rangle-\left\langle\left|D_{c}(\cdot)\right||x(k-2)|, w\right\rangle\right)+\cdots \\
& +\left(\left\langle\left|D_{c}(\cdot)\right||x(k-r+1)|, w\right\rangle-\left\langle\left|D_{c}(\cdot)\right||x(k-r)|, w\right\rangle\right) \\
= & \langle|x(k+1)|, w\rangle-\langle|x(k)|, w\rangle \\
& +\left(\left\langle\left|D_{c}(\cdot)\right||x(k)|, w\right\rangle-\left\langle\left|D_{c}(\cdot)\right||x(k-r)|, w\right\rangle\right) .
\end{aligned}
$$

Thus, by (3.12) we can obtain the following inequality:

$$
\begin{aligned}
\Delta v(x(k), k)< & \left\langle\left|A_{c}(\cdot)\right||x(k)|, w\right\rangle+\left\langle\left|D_{c}(\cdot)\right||x(k-r)|, w\right\rangle-\langle|x(k)|, w\rangle \\
& +\left(|| D_{c}(\cdot)|| x(k)|, w\rangle-\left\langle\left|D_{c}(\cdot)\right||x(k-r)|, w\right\rangle\right) .
\end{aligned}
$$

By some simple derivations, we have

$$
\begin{aligned}
\Delta v(k) & <\left\langle\left|A_{c}(\cdot)\right||x(k)|, w\right\rangle-\langle|x(k)|, w\rangle+\left\langle\left|D_{c}(\cdot)\right||x(k)|, w\right\rangle \\
& =\left\langle\left(\left|A_{c}(\cdot)\right|+\left|D_{c}(\cdot)\right|-I_{n}\right)|x(k)|, w\right\rangle=\left\langle\left(T_{c}(\cdot)-I_{n}\right)|x(k)|, w\right\rangle,
\end{aligned}
$$

where the matrix $T_{c}(\cdot)$ is defined in (3.1).

On the other hand, we assume that $\left(I_{n}-T_{c}(\cdot)\right)$ is an $M$-matrix, and according to the $M$-matrix proprieties, we can find a vector $\rho \in \Re_{+}^{* n}\left(\rho_{p} \in \Re_{+}^{*}, p=1, \ldots, n\right)$ satisfying the relation $\left(I_{n}-T_{c}(\cdot)\right)^{T} w=\rho, \forall w \in \Re_{+}^{* n}$. Then we can write

$$
\left\langle\left(I_{n}-T_{c}(\cdot)\right)|x(k)|, w\right\rangle=\left\langle\left(I_{n}-T_{c}(\cdot)\right)^{T} w,|x(k)|\right\rangle=\langle\rho,|x(k)|\rangle .
$$

This implies

$$
\left\langle\left(T_{c}(\cdot)-I_{n}\right)|x(k)|, w\right\rangle=\langle-\rho,|x(k)|\rangle .
$$


From the above derivations, we can obtain the following result:

$$
\Delta v(k) \leq\left\langle\left(T_{c}(\cdot)-I_{n}\right)|x(k)|, w\right\rangle \leq-\sum_{p=1}^{n} \rho_{p}\left|x_{p}(k)\right|<0
$$

Therefore, switched system (2.6) is robust asymptotically stable under switching law (2.3) and all admissible uncertainties (2.4) and (2.5). This completes the proof.

\section{Application to discrete-time uncertain switched nonlinear time-delay systems defined by difference equations}

In this section, to demonstrate the potential and validity of our developed results, we will presented an application for discrete-time uncertain switched nonlinear time-delay systems governed by the following switched nonlinear difference equation [31]:

$$
\begin{gathered}
y(k+n)+\sum_{i=1}^{N} \xi_{i}(k)\left(\sum_{l=1}^{N_{l}} \mu_{i l}(k) \sum_{p=0}^{n-1} a_{i l}^{n-p}(\cdot) y(k+p)\right. \\
\left.+\sum_{q=1}^{N_{q}} \lambda_{i q}(k) \sum_{p=0}^{n-1} d_{i q}^{n-p}(\cdot) y(k+p-\tau)\right)=0,
\end{gathered}
$$

where $\xi_{i}(k), i \in I[1 N]$ are the components of the switching function given in (2.3), $\mu_{i l}(k)$, $l \in I\left[1 N_{l}\right]$ and $\lambda_{i q}(k), q \in I\left[1 N_{q}\right]$ are unknown polytopic uncertain parameters given in (2.4) and (2.5) for each $i \in I[1 N]$.

Therefore, the presence of time-delay terms, the nonlinearities of coefficients, and unknown polytopic uncertain parameters makes the stability analysis for system (4.1) very difficult.

To solve this problem, the following change of variable will be adopted:

$$
x_{p+1}(k)=y(k+p), \quad p=0, \ldots, n-1 .
$$

Substituting relation (4.2) in equation (4.1), we obtain

$$
\begin{aligned}
x_{p}(k+1)= & x_{p+1}(k), \quad p=1, \ldots, n-1, \\
x_{n}(k+1)= & \sum_{i=1}^{N} \xi_{i}(k)\left(-\sum_{l=1}^{N_{l}} \mu_{i l}(k) \sum_{p=0}^{n-1} a_{i l}^{n-p}(\cdot) x_{p+1}(k)\right. \\
& \left.-\sum_{q=1}^{N_{q}} \lambda_{i q}(k) \sum_{p=0}^{n-1} d_{i l}^{n-p}(\cdot) x_{p+1}(k-\tau)\right)
\end{aligned}
$$

or under matrix representation, we have

$$
\left\{\begin{array}{l}
x(k+1)=\sum_{i=1}^{N} \xi_{i}(k)\left(\sum_{l=1}^{N_{l}} \mu_{i l}(k) A_{i l}(\cdot) x(k)+\sum_{q=1}^{N_{q}} \lambda_{i q}(k) D_{i q}(\cdot) x(k-\tau)\right), \\
x(s)=\phi(s), \quad s=-\tau, \ldots,-1,0
\end{array}\right.
$$

where $x(k)$ is the state vector of components $x_{p}(k), p=1, \ldots, n . \xi_{i}(k), i \in I[1 N]$ are the components of the switching function given in (2.3), $\mu_{i l}(k), l \in I\left[1 N_{l}\right]$ and $\lambda_{i q}(k), q \in I\left[1 N_{q}\right]$ are unknown polytopic uncertain parameters given in (2.4) and (2.5) for each $i \in I[1 N]$. 
From (4.3) and (4.4), it is clear that the vertex matrices $A_{i l}(\cdot), l \in I\left[1 N_{l}\right]$ and $D_{i q}(\cdot), q \in$ $I\left[1 N_{q}\right]$ for each $i \in I[1 N]$ are defined as follows:

$$
\begin{aligned}
A_{i l}(\cdot) & =\left[\begin{array}{cccc}
0 & 1 & \cdots & 0 \\
0 & 0 & \ddots & \vdots \\
\vdots & \vdots & \ddots & 1 \\
-a_{i l}^{n}(\cdot) & -a_{i l}^{n-1}(\cdot) & \cdots & -a_{i l}^{1}(\cdot)
\end{array}\right], \\
D_{i q}(\cdot) & =\left[\begin{array}{cccc}
0 & 0 & \cdots & 0 \\
0 & 0 & \ddots & \vdots \\
\vdots & \vdots & \ddots & 0 \\
-d_{i q}^{n}(\cdot) & -d_{i q}^{n-1}(\cdot) & \cdots & -d_{i q}^{1}(\cdot)
\end{array}\right],
\end{aligned}
$$

where $a_{i l}^{j}(\cdot)$ is a coefficient of the instantaneous characteristic polynomial $P_{i l}(\lambda), i \in I[1 N]$ and $l \in I\left[1 N_{l}\right]$ of the vertex matrix $A_{i l}(\cdot)$ which is given by

$$
P_{i l}(\lambda)=\lambda^{n}+\sum_{p=0}^{n-1} a_{i l}^{n-p}(\cdot) \lambda^{p}
$$

and $d_{i q}^{j}(\cdot)$ is a coefficient of the instantaneous characteristic polynomial $Q_{i q}(\lambda), i \in I[1 N]$ and $q \in I\left[1 N_{q}\right]$ of the vertex matrix $D_{i q}(\cdot)$ which is defined as follows:

$$
Q_{i q}(\lambda)=\sum_{p=0}^{n-1} d_{i q}^{n-p}(\cdot) \lambda^{p}
$$

To simplify the application to the Kotelyanski lemma, we consider a coordinate transformation into the arrow matrix form.

Therefore, to accomplish this aim, the following new state vector [31] will be considered:

$$
z(k)=P x(k)
$$

with

$$
P=\left[\begin{array}{ccccc}
1 & 1 & \ldots & 1 & 0 \\
\alpha_{1} & \alpha_{2} & \ldots & \alpha_{n-1} & 0 \\
\left(\alpha_{1}\right)^{2} & \left(\alpha_{2}\right)^{2} & \ldots & \left(\alpha_{n-1}\right)^{2} & \vdots \\
\vdots & \vdots & \ldots & \vdots & 0 \\
\left(\alpha_{1}\right)^{n-1} & \left(\alpha_{2}\right)^{n-1} & \ldots & \left(\alpha_{n-1}\right)^{n-1} & 1
\end{array}\right]
$$

where $\alpha_{j}(j=1, \ldots, n-1), \alpha_{j} \neq \alpha_{q}, \forall j \neq q$, are free real parameters that can be chosen arbitrarily.

Now, substituting (4.10) and (4.11) into (4.4) yields the following representation:

$$
\left\{\begin{array}{l}
z(k+1)=\sum_{i=1}^{N} \xi_{i}(k)\left(\sum_{l=1}^{N_{l}} \mu_{i l}(k) E_{i l}(\cdot) z(k)+\sum_{q=1}^{N_{q}} \lambda_{i q}(k) F_{i q}(\cdot) z(k-\tau)\right) \\
z(s)=P \phi(s), \quad s=-\tau, \ldots,-1,0
\end{array}\right.
$$


where $P$ is the corresponding passage matrix, $E_{i l}(\cdot), l \in I\left[1 N_{l}\right]$ and $F_{i q}(\cdot), q \in I\left[1 N_{q}\right]$ for each $i \in I[1 N]$ are vertex matrices in the arrow form given as follows:

$$
\begin{aligned}
E_{i l}(\cdot)=P^{-1} A_{i l}(\cdot) P & =\left[\begin{array}{ccccc}
\alpha_{1} & 0 & \cdots & 0 & \beta_{1} \\
0 & \ddots & \ddots & \vdots & \vdots \\
\vdots & \ddots & \ddots & 0 & \vdots \\
0 & \cdots & 0 & \alpha_{n-1} & \beta_{n-1} \\
\gamma_{i l}^{1}(\cdot) & \cdots & \cdots & \gamma_{i l}^{n-1}(\cdot) & \gamma_{i l}^{n}(\cdot)
\end{array}\right], \\
F_{i q}(\cdot)=P^{-1} D_{i q}(\cdot) P & =\left[\begin{array}{ccc}
0_{n-1, n-1} & 0_{n-1,1} \\
\delta_{i q}^{1}(\cdot) \cdots \delta_{i q}^{n-1}(\cdot) & \delta_{i q}^{n}(\cdot)
\end{array}\right] .
\end{aligned}
$$

Then, let us introduce the elements of the vertex matrices $E_{i l}(\cdot)$ such that

$$
\left\{\begin{array}{l}
\beta_{j}=\prod_{\substack{q=1 \\
q \neq j}}^{n-1}\left(\alpha_{j}-\alpha_{q}\right)^{-1}, \quad \forall j=1, \ldots, n-1, \\
\gamma_{i l}^{j}(\cdot)=-P_{i l}\left(\alpha_{j}\right), \quad \forall j=1, \ldots, n-1, \\
\gamma_{i l}^{n}(\cdot)=-a_{i l}^{1}(\cdot)-\sum_{j=1}^{n-1} \alpha_{j}
\end{array}\right.
$$

and the elements of the vertex matrices $F_{i q}(\cdot)$ are

$$
\left\{\begin{array}{l}
\delta_{i q}^{j}(\cdot)=-Q_{i q}\left(\alpha_{j}\right), \quad \forall j=1, \ldots, n-1 \\
\delta_{i q}^{n}(\cdot)=-d_{i q}^{1}(\cdot)
\end{array}\right.
$$

Due to the previous relations, the matrix $T_{i}(\cdot)$ for each $i \in I[1 N]$ will be defined as follows:

$$
T_{i}(\cdot)=\left[\begin{array}{ccccc}
\left|\alpha_{1}\right| & 0 & \cdots & 0 & \left|\beta_{1}\right| \\
0 & \ddots & \ddots & \vdots & \vdots \\
\vdots & \ddots & \ddots & 0 & \vdots \\
0 & \cdots & 0 & \left|\alpha_{n-1}\right| & \left|\beta_{n-1}\right| \\
t_{i}^{1}(\cdot) & \cdots & \cdots & t_{i}^{n-1}(\cdot) & t_{i}^{n}(\cdot)
\end{array}\right]
$$

with

$$
\left\{\begin{array}{l}
t_{i}^{j}(\cdot)=\left|\sum_{l=1}^{N_{l}} \mu_{i l}(k) \gamma_{i l}^{j}(\cdot)\right|+\left|\sum_{q=1}^{N_{q}} \lambda_{i q}(k) \delta_{i q}^{j}(\cdot)\right|, \quad j=1, \ldots, n-1, \\
t_{i}^{n}(\cdot)=\left|\sum_{l=1}^{N_{l}} \mu_{i l}(k) \gamma_{i l}^{n}(\cdot)\right|+\left|\sum_{q=1}^{N_{q}} \lambda_{i q}(k) \delta_{i q}^{n}(\cdot)\right| .
\end{array}\right.
$$

Then, the matrix $T_{\sigma(k)}(\cdot)$ is given as follows:

$$
T_{\sigma(k)}(\cdot)=\left[\begin{array}{ccccc}
\left|\alpha_{1}\right| & 0 & \cdots & 0 & \left|\beta_{1}\right| \\
0 & \ddots & \ddots & \vdots & \vdots \\
\vdots & \ddots & \ddots & 0 & \vdots \\
0 & \cdots & 0 & \left|\alpha_{n-1}\right| & \left|\beta_{n-1}\right| \\
\sum_{i=1}^{N} \xi_{i}(k) t_{i}^{1}(\cdot) & \cdots & \cdots & \sum_{i=1}^{N} \xi_{i}(k) t_{i}^{n-1}(\cdot) & \sum_{i=1}^{N} \xi_{i}(k) t_{i}^{n}(\cdot)
\end{array}\right]
$$


Now, after the above formulation, based on the Kotelyanski lemma, we are in a position to give sufficient stability conditions for system (4.5) that are presented in the following theorem.

Theorem 3 System (4.5) is globally robust asymptotically stable, for any arbitrary switching signal (2.3) and all admissible uncertainties (2.4) and (2.5), if there exist $\alpha_{j}(j=$ $1,2, \ldots, n-1), \alpha_{j} \neq \alpha_{q}, \forall j \neq q$, such that

(i) $1-\left|\alpha_{j}\right|>0, \quad j=1, \ldots n-1$,

(ii) $\quad 1-\left(\bar{t}^{n}(\cdot)\right)-\sum_{j=1}^{n-1}\left(\bar{t}^{j}(\cdot)\right)\left|\beta_{j}\right|\left(1-\left|\alpha_{j}\right|\right)^{-1}>0$

with

$$
\left\{\begin{array}{l}
\bar{t}^{n}(\cdot)=\max _{1 \leq i \leq N}\left(t_{i}^{n}(\cdot)\right), \\
\bar{t}^{j}(\cdot)=\max _{1 \leq i \leq N}\left(t_{i}^{j}(\cdot)\right), \quad j=1, \ldots, n-1 .
\end{array}\right.
$$

Proof For an arbitrary choice $\left|\alpha_{j}\right|<1, j=1, \ldots, n-1, \alpha_{j} \neq \alpha_{q}, \forall j \neq q$, we obtain the matrix $T_{c}(\cdot)$ as follows:

$$
T_{c}(\cdot)=\left[\begin{array}{ccccc}
\left|\alpha_{1}\right| & 0 & \cdots & 0 & \left|\beta_{1}\right| \\
0 & \ddots & \ddots & \vdots & \vdots \\
\vdots & \ddots & \ddots & 0 & \vdots \\
0 & \cdots & 0 & \left|\alpha_{n-1}\right| & \left|\beta_{n-1}\right| \\
\bar{t}^{1}(\cdot) & \cdots & \cdots & \bar{t}^{n-1}(\cdot) & \bar{t}^{n}(\cdot)
\end{array}\right]
$$

where the elements $\bar{t}^{j}(\cdot)$ are defined in (4.22).

From (4.23), it is clear that all the elements of $T_{c}(\cdot)$ are positive. Therefore, the application of the Kotelyanski lemma to the matrix $\left(I_{n}-T_{c}(\cdot)\right)$ enables us to conclude the stability of system (4.5).

Due to (2.7), the following sufficient robust asymptotic stability conditions are obtained:

$$
\left(I_{n}-T_{c}(\cdot)\right)\left(\begin{array}{cccc}
1 & 2 & \cdots & j \\
1 & 2 & \cdots & j
\end{array}\right)>0 \quad(j=1,2, \ldots, n)
$$

It is clear that for $j=1, \ldots, n-1$, condition (4.20) is verified as follows: $0<\left|\alpha_{j}\right|<1$.

Therefore, the last condition $j=n$ yields

$$
\operatorname{det}\left(I_{n}-T_{c}(\cdot)\right)=\left|\begin{array}{ccccc}
1-\left|\alpha_{1}\right| & 0 & \cdots & 0 & -\left|\beta_{1}\right| \\
0 & \ddots & \ddots & \vdots & \vdots \\
\vdots & \ddots & \ddots & 0 & \vdots \\
0 & \cdots & 0 & 1-\left|\alpha_{n-1}\right| & -\left|\beta_{n-1}\right| \\
-\bar{t}^{1}(\cdot) & \cdots & \cdots & -\bar{t}^{n-1}(\cdot) & 1-\bar{t}^{n}(\cdot)
\end{array}\right| .
$$

It follows that $1-\left(\bar{t}^{n}(\cdot)\right)-\sum_{j=1}^{n-1}\left(\bar{t}^{j}(\cdot)\right)\left|\beta_{j}\right|\left(1-\left|\alpha_{j}\right|\right)^{-1}>0$. 
This ends the proof of Theorem 3.

To simplify the use of the stability conditions, Theorem 3 can be reduced to the corollary below.

Corollary 1 If system (4.5) is robust asymptotically stable, for any arbitrary switching signal (2.3) and all admissible uncertainties (2.4) and (2.5), the following conditions are satisfied $\left.\forall \alpha_{j} \in\right] 01\left[(j=1, \ldots, n-1), \alpha_{j} \neq \alpha_{q}, \forall j \neq q\right.$, for each $i \in I[1 N], l \in I\left[1 N_{l}\right]$ and $q \in I\left[1 N_{q}\right]$ :
(i) $\beta_{j}\left(\sum_{l=1}^{N_{l}} \mu_{i l}(k) P_{i l}\left(\cdot, \alpha_{j}\right)+\sum_{q=1}^{N_{q}} \lambda_{i q}(k) Q_{i q}\left(\cdot, \alpha_{j}\right)\right)<0$,
(ii) $\left(\sum_{l=1}^{N_{l}} \mu_{i l}(k) P_{i l}(\cdot, \lambda=1)+\sum_{q=1}^{N_{q}} \lambda_{i q}(k) Q_{i q}(\cdot, \lambda=1)\right)>0$,
(iii) $\left(\sum_{l=1}^{N_{l}} \mu_{i l}(k) \gamma_{i l}^{n}(\cdot)+\sum_{q=1}^{N_{q}} \lambda_{i q}(k) \delta_{i q}^{n}(\cdot)\right)>0$.

Proof ([43]) Due to (4.21), we can formulate the following inequality easily:

$$
\begin{aligned}
& \max _{1 \leq i \leq N}\left(\left|\sum_{l=1}^{N_{l}} \mu_{i l}(k) \gamma_{i l}^{n}(\cdot)+\sum_{q=1}^{N_{q}} \lambda_{i q}(k) \delta_{i q}^{n}(\cdot)\right|\right) \\
& \quad+\sum_{j=1}^{n-1}\left(\max _{1 \leq i \leq N}\left(\left|\sum_{l=1}^{N_{l}} \mu_{i l}(k) \gamma_{i l}^{j}(\cdot)+\sum_{q=1}^{N_{q}} \lambda_{i q}(k) \delta_{i q}^{j}(\cdot)\right|\right)\right)\left|\beta_{j}\right|\left(1-\left|\alpha_{j}\right|\right)^{-1}<1 .
\end{aligned}
$$

This implies

$$
\begin{aligned}
1 & -\max _{1 \leq i \leq N}\left(\left|\sum_{l=1}^{N_{l}} \mu_{i l}(k) \gamma_{i l}^{n}(\cdot)+\sum_{q=1}^{N_{q}} \lambda_{i q}(k) \delta_{i q}^{n}(\cdot)\right|\right) \\
& -\sum_{j=1}^{n-1}\left(\max _{1 \leq i \leq N}\left(\left|\sum_{l=1}^{N_{l}} \mu_{i l}(k) \gamma_{i l}^{j}(\cdot)+\sum_{q=1}^{N_{q}} \lambda_{i q}(k) \delta_{i q}^{j}(\cdot)\right|\right)\right)\left|\beta_{j}\right|\left(1-\left|\alpha_{j}\right|\right)^{-1}>0 .
\end{aligned}
$$

Then we can easily deduce that the relation below is more restrictive than relation (4.30) for each $i \in I[1 N]$ :

$$
\begin{aligned}
1 & -\left(\left|\sum_{l=1}^{N_{l}} \mu_{i l}(k) \gamma_{i l}^{n}(\cdot)+\sum_{q=1}^{N_{q}} \lambda_{i q}(k) \delta_{i q}^{n}(\cdot)\right|\right) \\
& -\sum_{j=1}^{n-1}\left(\left|\sum_{l=1}^{N_{l}} \mu_{i l}(k) \gamma_{i l}^{j}(\cdot)+\sum_{q=1}^{N_{q}} \lambda_{i q}(k) \delta_{i q}^{j}(\cdot)\right|\right)\left|\beta_{j}\right|\left(1-\left|\alpha_{j}\right|\right)^{-1}>0 .
\end{aligned}
$$

Now, by considering relations (4.26) and (4.28) in Corollary 1 and substituting (4.15), (4.16) into (4.31), we obtain (4.27). 
Then, to accomplish this aim, relation (4.31) becomes

$$
\begin{aligned}
1 & -\left(\left(\sum_{l=1}^{N_{l}} \mu_{i l}(k) \gamma_{i l}^{n}(\cdot)+\sum_{q=1}^{N_{q}} \lambda_{i q}(k) \delta_{i q}^{n}(\cdot)\right)\right) \\
& -\sum_{j=1}^{n-1}\left(\left(\sum_{l=1}^{N_{l}} \mu_{i l}(k) \gamma_{i l}^{j}(\cdot)+\sum_{q=1}^{N_{q}} \lambda_{i q}(k) \delta_{i q}^{j}(\cdot)\right)\right) \beta_{j}\left(1-\left|\alpha_{j}\right|\right)^{-1}>0 .
\end{aligned}
$$

It follows that

$$
\begin{aligned}
1 & +\left(\sum_{l=1}^{N_{l}} \mu_{i l}(k) a_{i l}^{1}(\cdot)+\sum_{q=1}^{N_{q}} \lambda_{i q}(k)(\cdot) d_{i q}^{1}(\cdot)\right) \\
& +\sum_{j=1}^{n-1} \alpha_{j}+\sum_{j=1}^{n-1}\left(\frac{1}{\left(1-\alpha_{j}\right)}\left(\frac{\left(\lambda-\alpha_{j}\right)\left(\sum_{l=1}^{N_{l}} \mu_{i l}(k) P_{i l}+\sum_{q=1}^{N_{q}} \lambda_{i q}(k)(\cdot) Q_{i q}\right)}{H(\lambda)}\right)\right)_{\lambda=\alpha_{j}}>0
\end{aligned}
$$

with

$$
H(\lambda)=\prod_{j=1}^{n-1}\left(\lambda-\alpha_{j}\right)
$$

To complete this proof, let us first simply see that

$$
\begin{aligned}
\frac{\left(\sum_{l=1}^{N_{l}} \mu_{i l}(k) P_{i l}(\cdot, \lambda)+\sum_{q=1}^{N_{q}} \lambda_{i q}(k)(\cdot) Q_{i q}(\cdot, \lambda)\right)}{H(\lambda)} \\
=\lambda+\left(\sum_{l=1}^{N_{l}} \mu_{i l}(k) a_{i l}^{1}(\cdot)+\sum_{q=1}^{N_{q}} \lambda_{i q}(k)(\cdot) d_{i q}^{1}(\cdot)\right) \\
\quad+\sum_{j=1}^{n-1} \alpha_{j}+\sum_{j=1}^{n-1}\left(\frac{\left(\lambda-\alpha_{j}\right)\left(\sum_{l=1}^{N_{l}} \mu_{i l}(k) P_{i l}(\cdot, \lambda)+\sum_{q=1}^{N_{q}} \lambda_{i q}(k)(\cdot) Q_{i q}(\cdot, \lambda)\right)}{\left(1-\alpha_{j}\right) H(\lambda)}\right)_{\lambda=\alpha_{j}} .
\end{aligned}
$$

From (4.33), (4.34) and (4.35), we can deduce that

$$
\left(\frac{\sum_{l=1}^{N_{l}} \mu_{i l}(k) P_{i l}(\cdot, \lambda)+\sum_{q=1}^{N_{q}} \lambda_{i q}(k)(\cdot) Q_{i q}(\cdot, \lambda)}{H(\lambda)}\right)_{\lambda=1}>0 .
$$

By a simple verification, we can get

$$
\left.H(\lambda=1)=\prod_{j=1}^{n-1}\left(1-\alpha_{j}\right)>0, \quad \forall \alpha_{j} \in\right] 01[.
$$

And finally, substituting (4.37) into the foregoing (4.36), gives

$$
\left(\sum_{l=1}^{N_{l}} \mu_{i l}(k) P_{i l}(\cdot, \lambda=1)+\sum_{q=1}^{N_{q}} \lambda_{i q}(k) Q_{i q}(\cdot, \lambda=1)\right)>0,
$$

which indicates that condition (4.27) is satisfied. 
Robust asymptotical stability of system (4.5) under any arbitrary switching law (2.3) and all admissible uncertainties (2.4) and (2.5) follows. This completes the proof.

\section{An illustrative example}

In this section, as an application of our results, let us consider system (4.1) with two subsystems described by the following switched difference equation:

$$
\begin{aligned}
y(k+2)= & \sum_{i=1}^{2} \xi_{i}(k)\left(\sum_{l=1}^{2} \mu_{i l}(k) \sum_{p=0}^{1} a_{i l}^{2-p}(\cdot) y(k+p)\right. \\
& \left.+\sum_{q=1}^{2} \lambda_{i q}(k) \sum_{p=0}^{1} d_{i q}^{2-p}(\cdot) y(k+p-\tau)\right)=0,
\end{aligned}
$$

where $\tau$ is the time-delay, $a_{i l}(\cdot)$ and $d_{i q}(\cdot)$ are nonlinear coefficients, $\mu_{i l}(k)$ and $\lambda_{i q}(k)$ are unknown polytopic uncertain parameters for each $i \in I[12], l \in I[12]$ and $q \in I[12] . \xi_{i}(k)$ is the arbitrary switching rule given in (2.3).

By (4.2), (4.3) and (4.5) this system will be given in the state form defined as follows:

$$
\left\{\begin{array}{l}
x(k+1)=\sum_{i=1}^{2} \xi_{i}(k)\left(\sum_{l=1}^{2} \mu_{i l}(k) A_{i l}(\cdot) x(k)+\sum_{q=1}^{2} \lambda_{i q}(k) D_{i q}(\cdot) x(k-\tau)\right), \\
x(s)=\phi(s), \quad s=-\tau, \ldots,-1,0
\end{array}\right.
$$

where the vertex matrices and the parameters are listed below:

$$
\begin{aligned}
& A_{11}(\cdot)=\left[\begin{array}{cc}
0 & 1 \\
-0.5+0.75 f(\cdot) & 1-0.5 f(\cdot)
\end{array}\right], \\
& A_{12}(\cdot)=\left[\begin{array}{cc}
0 & 1 \\
-0.6+0.8 f(\cdot) & 0.9-0.4 f(\cdot)
\end{array}\right], \\
& A_{21}(\cdot)=\left[\begin{array}{cc}
0 & 1 \\
-0.4+0.85 f(\cdot) & 1-0.5 f(\cdot)
\end{array}\right], \\
& A_{22}(\cdot)=\left[\begin{array}{cc}
0 & 1 \\
-0.5+0.7 f(\cdot) & 1.2-0.8 f(\cdot)
\end{array}\right], \\
& D_{11}(\cdot)=\left[\begin{array}{cc}
0 & 0 \\
-0.17+0.25 \Phi(\cdot) & 0.703-0.5 \Phi(\cdot)
\end{array}\right], \\
& D_{12}(\cdot)=\left[\begin{array}{cc}
0 & 0 \\
-0.2+0.2 \Phi(\cdot) & 0.87-0.6 \Phi(\cdot)
\end{array}\right], \\
& D_{21}(\cdot)=\left[\begin{array}{cc}
0 & 0.9-0.7 \Phi(\cdot)
\end{array}\right],
\end{aligned}
$$

and

$$
D_{22}(\cdot)=\left[\begin{array}{cc}
0 & 0 \\
-0.32+0.3 \Phi(\cdot) & 0.75-0.45 \Phi(\cdot)
\end{array}\right]
$$

where $f(\cdot)$ and $\Phi(\cdot)$ are general nonlinear functions. 
Let us introduce unknown uncertainty parameters satisfying the following conditions: $\mu_{11}(\cdot)=\lambda_{11}(\cdot)=\rho(\cdot), \mu_{12}(\cdot)=\lambda_{12}(\cdot)=1-\rho(\cdot), \mu_{21}(\cdot)=\lambda_{21}(\cdot)=\rho(\cdot)$ and $\mu_{22}(\cdot)=\lambda_{22}(\cdot)=1-$ $\rho(\cdot)$ with $\rho(\cdot)$ being a general nonlinearity such that $0 \leq \rho(\cdot) \leq 1$.

Now, due to (4.10), (4.11), (4.12), (4.13), (4.14), (4.15) and (4.16), the vertex matrices in arrow form are the following:

$$
\begin{aligned}
& E_{11}(\cdot)=\left[\begin{array}{cc}
\alpha & 1 \\
\gamma_{11}^{1}(\cdot) & \gamma_{11}^{2}(\cdot)
\end{array}\right], \quad E_{12}(\cdot)=\left[\begin{array}{cc}
\alpha & 1 \\
\gamma_{12}^{1}(\cdot) & \gamma_{12}^{2}(\cdot)
\end{array}\right], \quad E_{21}(\cdot)=\left[\begin{array}{cc}
\alpha & 1 \\
\gamma_{21}^{1}(\cdot) & \gamma_{21}^{2}(\cdot)
\end{array}\right] \\
& E_{22}(\cdot)=\left[\begin{array}{cc}
\alpha & 1 \\
\gamma_{22}^{1}(\cdot) & \gamma_{22}^{2}(\cdot)
\end{array}\right], \quad F_{11}(\cdot)=\left[\begin{array}{cc}
0 & 0 \\
\delta_{11}^{1}(\cdot) & \delta_{11}^{2}(\cdot)
\end{array}\right], \quad F_{12}(\cdot)=\left[\begin{array}{cc}
0 & 0 \\
\delta_{12}^{1}(\cdot) & \delta_{12}^{2}(\cdot)
\end{array}\right] \text {, } \\
& F_{21}(\cdot)=\left[\begin{array}{cc}
0 & 0 \\
\delta_{21}^{1}(\cdot) & \delta_{21}^{2}(\cdot)
\end{array}\right] \text { and } F_{22}(\cdot)=\left[\begin{array}{cc}
0 & 0 \\
\delta_{22}^{1}(\cdot) & \delta_{22}^{2}(\cdot)
\end{array}\right]
\end{aligned}
$$

with the following parameters:

$$
\begin{aligned}
& \left\{\begin{array}{l}
\gamma_{11}^{1}(\cdot)=-P_{11}(\alpha)=-\left[\alpha^{2}+(-1+0.5 f(\cdot)) \alpha+0.5-0.75 f(\cdot)\right], \\
\gamma_{11}^{2}(\cdot)=-(-1+0.5 f(\cdot)+\alpha),
\end{array}\right. \\
& \left\{\begin{array}{l}
\gamma_{12}^{1}(\cdot)=-P_{12}(\alpha)=-\left[\alpha^{2}+(-0.9+0.4 f(\cdot)) \alpha+0.6-0.8 f(\cdot)\right], \\
\gamma_{12}^{2}(\cdot)=-(-0.9+0.4 f(\cdot)+\alpha),
\end{array}\right. \\
& \left\{\begin{array}{l}
\gamma_{21}^{1}(\cdot)=-P_{21}(\alpha)=-\left[\alpha^{2}+(-1+0.5 f(\cdot)) \alpha+0.4-0.85 f(\cdot)\right], \\
\gamma_{21}^{2}(\cdot)=-(-1+0.5 f(\cdot)+\alpha),
\end{array}\right. \\
& \left\{\begin{array}{l}
\gamma_{22}^{1}(\cdot)=-P_{22}(\alpha)=-\left[\alpha^{2}+(-1.2+0.8 f(\cdot)) \alpha+0.5-0.7 f(\cdot)\right] \\
\gamma_{22}^{2}(\cdot)=-(-1.2+0.8 f(\cdot)+\alpha)
\end{array}\right. \\
& \left\{\begin{array}{l}
\delta_{11}^{1}(\cdot)=-Q_{11}(\alpha)=-[(-0.703+0.5 \Phi(\cdot)) \alpha+0.17-0.25 \Phi(\cdot)], \\
\delta_{11}^{2}(\cdot)=-(-0.703+0.5 \Phi(\cdot)),
\end{array}\right. \\
& \left\{\begin{array}{l}
\delta_{12}^{1}(\cdot)=-Q_{12}(\alpha)=-[(-0.87+0.6 \Phi(\cdot)) \alpha+0.2-0.2 \Phi(\cdot)], \\
\delta_{12}^{2}(\cdot)=-(-0.87+0.6 \Phi(\cdot)),
\end{array}\right. \\
& \left\{\begin{array}{l}
\delta_{21}^{1}(\cdot)=-Q_{21}(\alpha)=-[(-0.9+0.7 \Phi(\cdot)) \alpha+0.25-0.4 \Phi(\cdot)], \\
\delta_{21}^{2}(\cdot)=-(-0.9+0.7 \Phi(\cdot)),
\end{array}\right.
\end{aligned}
$$

and

$$
\left\{\begin{array}{l}
\delta_{22}^{1}(\cdot)=-Q_{22}(\alpha)=-[(-0.75+0.45 \Phi(\cdot)) \alpha-0.32+0.3 \Phi(\cdot)] \\
\delta_{22}^{2}(\cdot)=-(-0.75+0.45 \Phi(\cdot)) .
\end{array}\right.
$$

Then, by applying Corollary 1 , with $\alpha=0.2$ and $\beta=1$, we deduce the following stability conditions:

(i) $0<\alpha<1$,

(ii) $\rho(\cdot)\left(P_{11}(\alpha)+Q_{11}(\alpha)\right)+(1-\rho(\cdot))\left(P_{12}(\alpha)+Q_{12}(\alpha)\right)<0$,

(iii) $\rho(\cdot)\left(P_{21}(\alpha)+Q_{21}(\alpha)\right)+(1-\rho(\cdot))\left(P_{22}(\alpha)+Q_{22}(\alpha)\right)<0$,

(iv) $\rho(\cdot)\left(P_{11}(1)+Q_{11}(1)\right)+(1-\rho(\cdot))\left(P_{12}(1)+Q_{12}(1)\right)>0$,

(v) $\rho(\cdot)\left(P_{21}(1)+Q_{21}(1)\right)+(1-\rho(\cdot))\left(P_{22}(1)+Q_{22}(1)\right)>0$, 


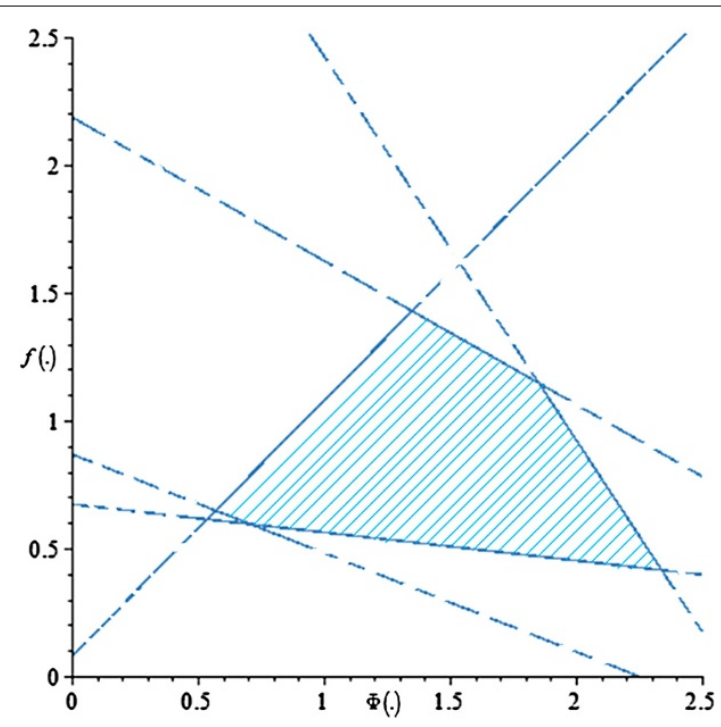

Figure 1 Robust stability domain obtained from Corollary $1: \rho(\cdot)=0$.

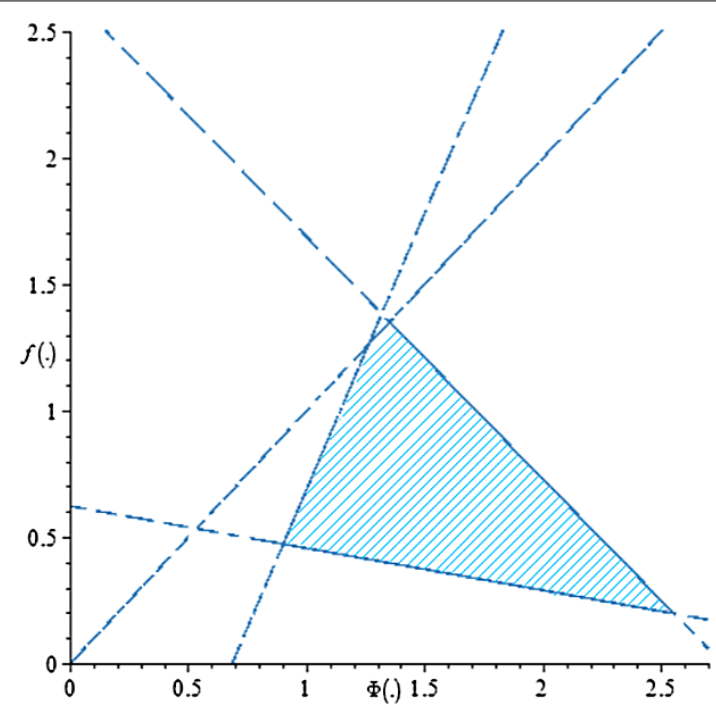

Figure 2 Robust stability domain obtained from Corollary $1: \rho(\cdot)=0.5$.

(vi) $\rho(\cdot)\left(\gamma_{11}^{2}(\cdot)+\delta_{11}^{2}\right)+(1-\rho(\cdot))\left(\gamma_{12}^{2}(\cdot)+\delta_{12}^{2}\right)>0$,

(vii) $\rho(\cdot)\left(\gamma_{21}^{2}(\cdot)+\delta_{21}^{2}\right)+(1-\rho(\cdot))\left(\gamma_{22}^{2}(\cdot)+\delta_{22}^{2}\right)>0$.

Thus, by (ii), (iii), (iv), (v), (vi) and (vii) we obtain:

(i) $\left(\alpha^{2}-1.77 \alpha+0.8+f(\cdot)(0.4 \alpha-0.8)+\Phi(\cdot)(0.6 \alpha-0.2)\right)+(\rho(\cdot))(-0.067 \alpha-0.13+$ $f(\cdot)(0.1 \alpha-0.05)+\Phi(\cdot)(-0.1 \alpha-0.05))<0$,

(ii) $\left(\alpha^{2}-1.95 \alpha+0.82+f(\cdot)(0.8 \alpha-0.7)+\Phi(\cdot)(0.45 \alpha-0.3)\right)+(\rho(\cdot))(0.05 \alpha-0.17+$ $f(\cdot)(-0.3 \alpha-0.15)+\Phi(\cdot)(0.35 \alpha-0.1))<0$,

(iii) $(-0.4 f(\cdot)+0.4 \Phi(\cdot)+0.03)+(\rho(\cdot))(0.15 f(\cdot)-0.15 \Phi(\cdot)-0.063)>0$,

(iv) $(0.1 f(\cdot)+0.15 \Phi(\cdot)-0.13)+(\rho(\cdot))(-0.45 f(\cdot)+0.25 \Phi(\cdot)-0.12)>0$,

(v) $(-0.4 f(\cdot)-0.6 \Phi(\cdot)+1.77-\alpha)+(\rho(\cdot))(-0.1 f(\cdot)+0.1 \Phi(\cdot)-0.067)>0$,

(vi) $(-0.8 f(\cdot)-0.45 \Phi(\cdot)+1.95-\alpha)+(\rho(\cdot))(0.3 f(\cdot)-0.35 \Phi(\cdot)-0.05)>0$. 


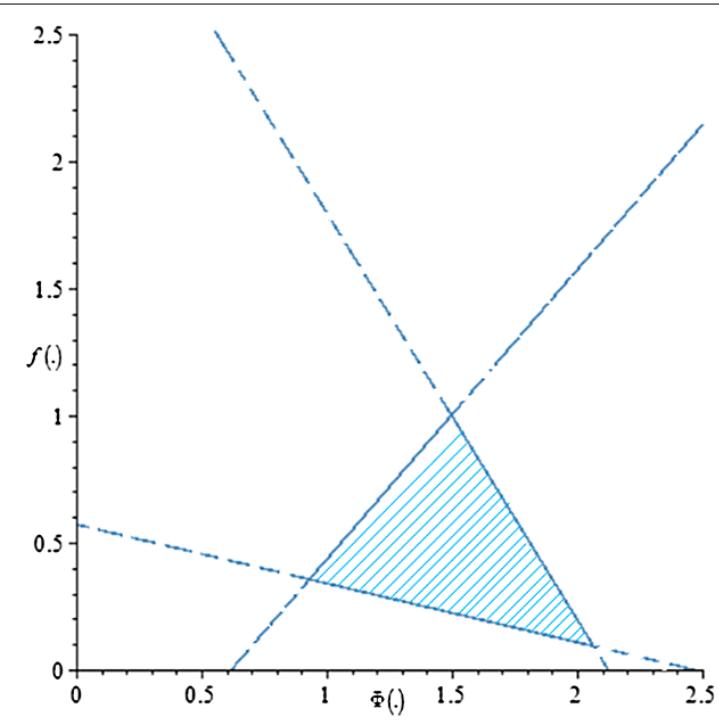

Figure 3 Robust stability domain obtained from Corollary $1: \rho(\cdot)=1$.

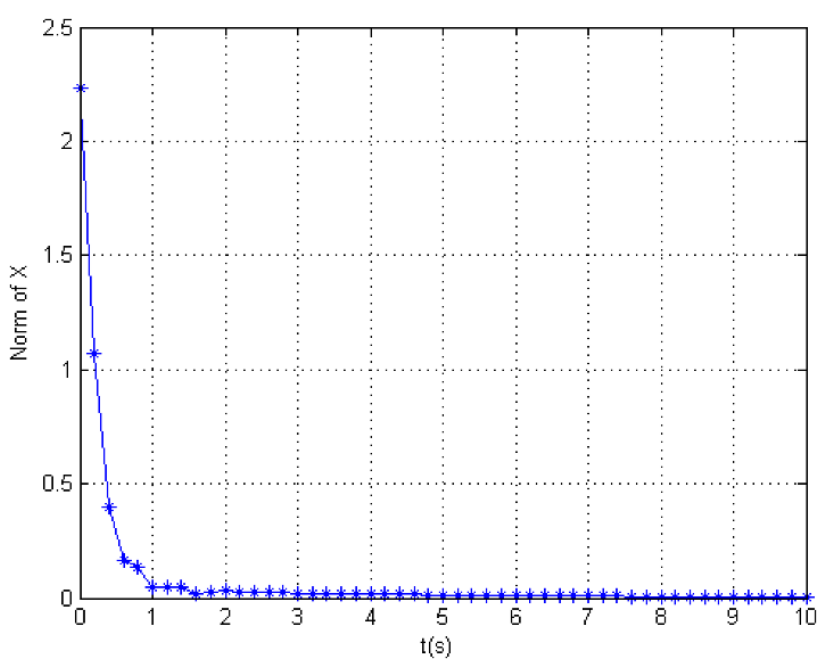

Figure 4 The state's norm of the switched system in the example with $t_{1}=k_{1} T_{e}=0.4 \mathrm{~s}$.

Due to those inequalities, the robust stability domains given by the nonlinear $f(\cdot)$ relative to the nonlinear $\Phi(\cdot)$ for different values chosen of the nonlinear incertitude parameter $\rho(\cdot)=0,0.5$ and 1 are illustrated in Figure 1, Figure 2 and Figure 3, respectively.

Based on these typical results plotted in Figures 1-3, we can see that the stability domains are closely related to the values taken by the uncertainty parameters.

Now, according to the previous robust stability domains, for particular values chosen of the uncertain parameter $\rho(\cdot)=0.5$, the nonlinearities $f(\cdot)=0.9$ and $\Phi(\cdot)=1.7$. The simulation results are obtained with sampling period $T_{e}=0.2 \mathrm{~s}$. We suppose that the vectorvalued initial function $\phi(s)=\left[\begin{array}{ll}1 & 2\end{array}\right]^{T}$ for all $s=-1,0$. According to the switched law (3), the final time $t_{f}=k T_{e}=10 \mathrm{~s}$ and the switched time $t_{1}=k_{1} T_{e}=0.4 \mathrm{~s}$, a typical result is plotted in Figure 4, Figure 5 and Figure 6 which show the norm of the state, the system state and the state space converge to zero. 


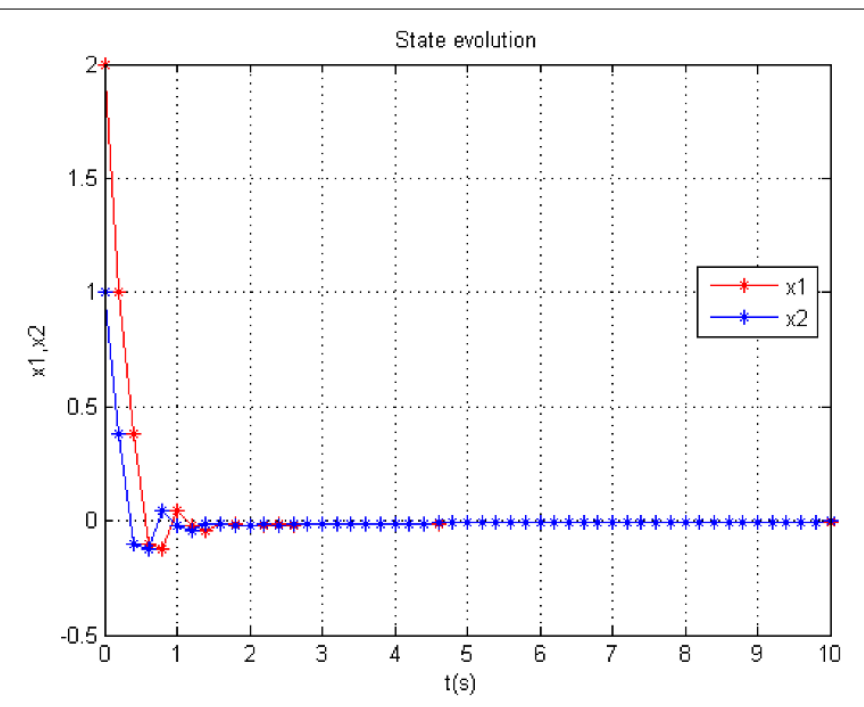

Figure 5 The state response of the switched system in the example with $t_{1}=k_{1} T_{e}=0.4 \mathrm{~s}$.

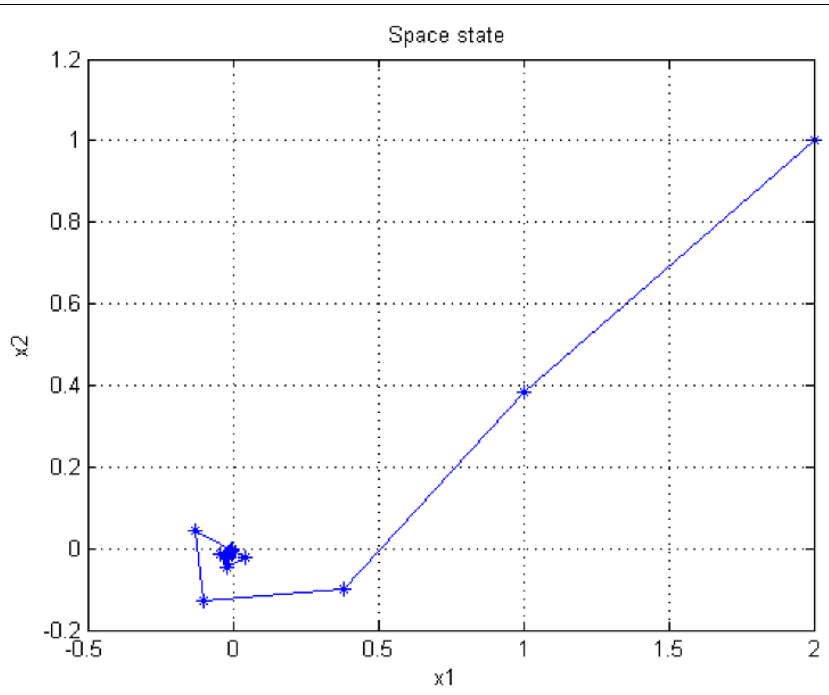

Figure 6 The state of the switched system in the example with $t_{1}=k_{1} T_{e}=0.4 \mathrm{~s}$.

It can be seen from Figures 4-6 that the system is stable, which demonstrates the effectiveness of the proposed method.

Next, we study the case that we have the same values chosen of the uncertain parameter $\rho(\cdot)=0.5$, the nonlinearities $f(\cdot)=0.9$ and $\Phi(\cdot)=1.7$, and the sampling time $T_{e}=0.2 s$. The switched time $t_{1}=k_{1} T_{e}=5 s$, and we suppose that the initial state vector is $\phi(s)=\left[\begin{array}{ll}1 & 2\end{array}\right]^{T}$ for all $s=-5,-4,-3,-2,-1,0$. The norm of the state, evolution of the states and the state space are shown in Figure 7, Figure 8 and Figure 9, respectively.

Therefore, Figures 7-9 allow to conclude that the switched system converges to zero.

This example was used to illustrate the effectiveness of our developed approach with different values of delay and different switched time. Besides, this example shows that the obtained stability conditions are sufficient and very close to be necessary. 


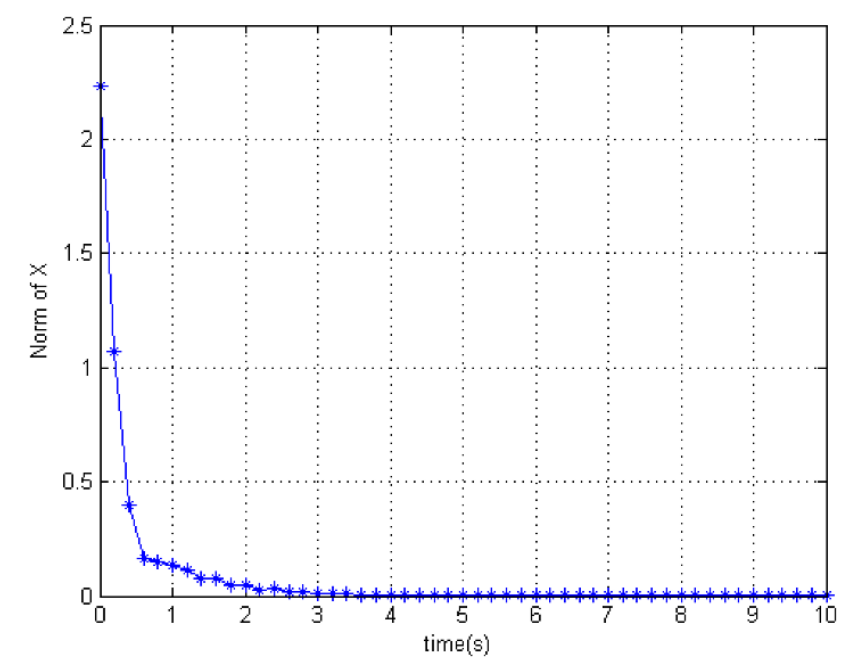

Figure 7 The state's norm of the switched system in the example with $t_{1}=k_{1} T_{e}=5 \mathrm{~s}$.

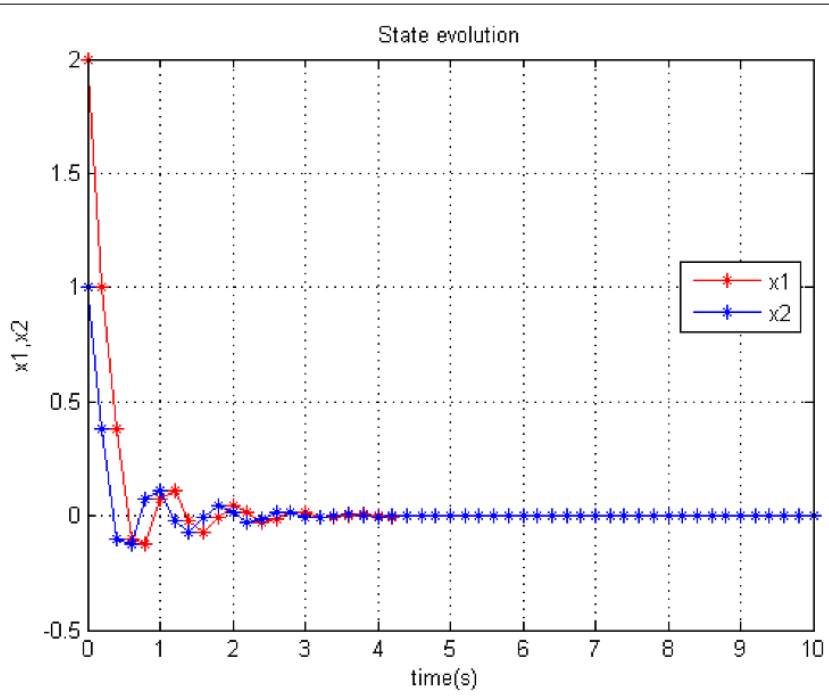

Figure 8 The state response of the switched system in the example with $t_{1}=k_{1} T_{e}=5 \mathrm{~s}$.

It is should be noted that this proposed approach is less conservative that searching for a common Lyapunov function. In fact, in [37] the authors introduced a simple linear example without time-delay for which a common Lyapunov function does not exist. Therefore, we cannot guarantee stability under arbitrary switching.

\section{Conclusions}

This paper has investigated new robust delay-independent stability conditions for a class of discrete-time switched nonlinear time-delay systems with polytopic uncertainties. These stability conditions were deduced with the help of the construction of an appropriated common Lyapunov function, and also by the resort to the Kotelyanski lemma and the $M$-matrix proprieties. 


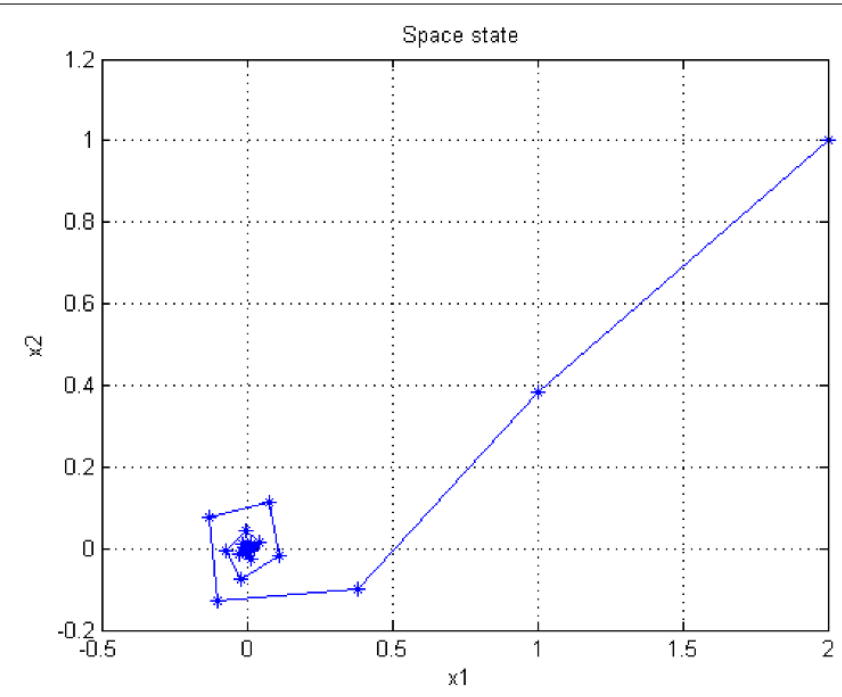

Figure 9 The state of the switched system in the example with $t_{1}=k_{1} T_{e}=5 \mathrm{~s}$.

Compared with the existing results of switched systems, these obtained results are formulated in terms of the uncertain parameters, explicit and easy to apply. Moreover, this method allows us to avoid searching for a common Lyapunov function. A numerical example is given to show the effectiveness of our proposed approach.

This proposed approach could be further used as a constructive solution to the problems of state and static output feedback stabilization.

The limits of this paper are that it has been confined to the boundaries of numerical examples. It would be beneficial to extend the research further so as to include real systems and to conduct a comparative study with an example for which a common Lyapunov function exists.

\section{Competing interests}

The authors declare that they have no competing interests.

\section{Authors' contributions}

The authors contributed equally to the writing of this paper. All authors read and approved the final manuscript.

\section{Acknowledgements}

The authors would like to thank the referees and the editor for helpful suggestions incorporated into this paper.

Received: 13 March 2014 Accepted: 4 August 2014 Published: 09 Sep 2014

\section{References}

1. Mahmoud, S: Switched Time-Delay Systems. Springer, Boston (2010)

2. Liberzon, D: Switching in Systems and Control. Springer, Boston (2003)

3. Li, Z, Soh, Y, Wen, C: Switched and Impulsive Systems: Analysis, Design and Applications. Springer, Berlin (2005)

4. Yu, M, Wang, L, Chu, TG, Xie, GM: Stabilization of networked control systems with data packet dropout and network delays via switching system approach. In: Proc. the 43th IEEE Conf. Decision and Control, vol. 4, pp. 3545-3550 (2004)

5. Donkers, M, Heemels, W, Wouw, N, Hetel, L: Stability analysis of networked control systems using a switched linear systems approach. IEEE Trans. Autom. Control 56, 2101-2115 (2011)

6. Gans, NR, Hutchinson, SA: Stable visual servoing through hybrid switched-system control. IEEE Trans. Robot. 23 530-540 (2007)

7. Narendra, KS, Balakrishnan, J: A common Lyapunov function for stable LTI systems with commuting A-matrices. IEEE Trans. Autom. Control 39, 2469-2471 (1994)

8. Shim, H, Noh, DJ, Seo, JH: Common Lyapunov function for exponentially stable nonlinear systems. J. Korean Inst. Electr. Eng. 11, 108-111 (2001)

9. Vu, L, Liberzon, D: Common Lyapunov functions for families of commuting nonlinear systems. Syst. Control Lett. 54 405-416 (2005) 
10. Branicky, MS: Multiple Lyapunov functions and other analysis tools for switched and hybrid systems. IEEE Trans. Autom. Control 43, 475-482 (1998)

11. Ishii, H, Francis, BA: Stabilizing a linear system by switching control with dwell time. IEEE Trans. Autom. Control 47, 1962-1973 (2002)

12. Hespanha, JP, Morse, AS: Stability of switched systems with average dwell-time. In: 38th IEEE Conf. Decision and Control, Phoenix (1999)

13. Daafouz, J, Riedinger, P, lung, C: Stability analysis and control synthesis for switched systems: a switched Lyapunov function approach. IEEE Trans. Autom. Control 47, 1883-1887 (2002)

14. Chang, L, Ker, Y, Jenq-Der, C, Long-Yeu, C: Sufficient conditions for global exponential stability of discrete switched time-delay systems with linear fractional perturbations via switching signal design. Adv. Differ. Equ. 2013, 39 (2013)

15. Jianyin, F, Kai, L: Stabilization of nonlinear systems under arbitrary switching law. In: Proceedings of the 30th Chinese Control Conference, Beijing, China (2010)

16. Zhang, WA, Yu, L: Stability analysis for discrete-time switched time-delay systems. Automatica 45, $2265-2271$ (2009)

17. Lien, CH, Yu, KW, Chang, HC, Chung, LY, Chen, JD: Switching signal design for exponential stability of discrete switched systems with interval time varying delay. J. Franklin Inst. 349, 2182-2192 (2012)

18. Sangapate, P: New sufficient conditions for the asymptotic stability of discrete time-delay systems. Adv. Differ. Equ. 2012, 28 (2012)

19. Sun, YG, Wang, L, Xie, GM: Delay-dependent robust stability and stabilization for discrete-time switched systems with mode-dependent time-varying delays. Appl. Math. Comput. 180, 428-435 (2006)

20. Lien, CH, Yu, KW, Chung, YJ, Chang, HC, Chung, LY, Chen, JD: Switching signal design for global exponential stability of uncertain switched nonlinear systems with time-varying delay. Nonlinear Anal. Hybrid Syst. 5, 10-19 (2011)

21. Dong, Y, Wei, J: Output feedback stabilization of nonlinear discrete-time systems with time-delay. Adv. Differ. Equ. 2012, $73(2012)$

22. Zhang, WA, Yu, L: Stability analysis for discrete-time switched time-delay systems. Automatica 45(10), 2265-2271 (2009)

23. Sun, XM, Wang, W, Liu, GP, Zhao, J: Stability analysis for linear switched systems with time-varying delay. IEEE Trans. Syst. Man Cybern., Part B, Cybern. 38, 528-533 (2008)

24. Zhang, L, Shi, P, Basin, M: Robust stability and stabilization of uncertain switched linear discrete time-delay systems. IET Control Theory Appl. 2, 606-614 (2008)

25. Zhai, G, Lin, H, Antsaklis, PJ: Quadratic stabilizability of switched linear systems with polytopic uncertainties. Int. J. Control 76, 747-753 (2003)

26. Lin, H, Antsaklis, PJ: Switching stabilizability for continuous-time uncertain switched linear systems. IEEE Trans. Autom. Control 52,633-646 (2007)

27. Sun, Z, Ge, SS: Switched Linear Systems Control and Design. Springer, London (2005)

28. Ibrir, S: Stability and robust stabilization of discrete-time switched systems with time-delays: LMI approach. Appl. Math. Comput. 206, 570-578 (2008)

29. Liu, J, Liu, X, Xie, WC: Delay-dependent robust control for uncertain switched systems with time-delay. Nonlinear Anal. Hybrid Syst. 2, 81-95 (2008)

30. Sun, YG, Wang, L, Xie, G: Delay-dependent robust stability and $H \infty$ control for uncertain discrete-time switched systems with mode-dependent time delays. Appl. Math. Comput. 187, 1228-1237 (2007)

31. Kermani, M, Jaballi, A, Sakly, A: A new stability analysis for discrete-time switched time-delay systems. Int. J. Control Energy Electr. Eng. 1, 23-30 (2014)

32. Kermani, M, Sakly, A, M'Sahli, F: A new stability analysis and stabilization of discrete-time switched linear systems using vector norms approach. World Acad. Sci., Eng. Technol. 71, 1302-1307 (2012)

33. Kermani, M, Sakly, A, M'Sahli, F: A new stability analysis and stabilization of uncertain switched linear systems based on vector norms approach. In: 10th International Multi-Conference on Systems, Signals \& Devices (SSD), Hammamet, Tunisia (2013)

34. Xie, D, Xu, G: Computation of performance robustness bounds for control systems with parameter uncertainties: an LMI approach. IEE Proc., Control Theory Appl. 152, 675-682 (2005)

35. Chen, N, Gui, W, Liu, B: Parametric absolute stability of interconnected Lurie control systems. Acta Autom. Sin. 33, 1283-1289 (2007)

36. Zhanga, X, Gaoc, Y, Xia, Z: Stabilization of switched systems with polytopic uncertainties via composite quadratic functions. Nonlinear Anal. Hybrid Syst. 11, 71-83 (2014)

37. Minh, VT: Stability for switched dynamic hybrid systems. Math. Comput. Model. 57, 78-83 (2013)

38. Shatyrko, A, Diblik, J, Khusainov, D, Ruzickova, M: Stabilization of Lur'e-type nonlinear control systems by Lyapunov-Krasovski functionals. Adv. Differ. Equ. (2012). doi:10.1186/1687-2012-229

39. Khusainov, D, Shatyrko, A: Absolute stability conditions for difference systems. Bull. Taras Shevchenko Natl. Univ. Kyiv Cybern. 10, 34-48 (2010)

40. Elmadssia, S, Saadaoui, K, Benrejeb, M: New delay-dependent stability conditions for linear systems with delay. In: International Conference on Communications, Computing and Control Applications (CCCA), Hammamet, Tunisia (2011)

41. Elmadssia, S, Saadaoui, K, Benrejeb, M: New delay-dependent stability conditions for linear systems with delay. Syst. Sci. Control Eng. 1, 37-41 (2013)

42. Benrejeb, M, Soudani, D, Sakly, A, Borne, P: New discrete Tanaka Sugeno Kang fuzzy systems characterization and stability domain. Int. J. Comput. Commun. Control 1, 9-19 (2006)

43. Sfaihi, B, Benrejeb, M: On stability analysis of nonlinear discrete singularly perturbed T-S fuzzy models. Int. J. Dyn. Control 1, 20-31 (2013)

44. Benrejeb, M, Borne, P: On an algebraic stability criterion for non-linear processes. Interpretation in the frequency domain. In: Proceedings of the Measurement and Control International Symposium MECO'78, Athens (1978)

45. Benrejeb, M, Gasmi, M: On the use of an arrow form matrix for modelling and stability analysis of singularly perturbed nonlinear systems. Syst. Anal. Model. Simul. 40, 509-525 (2001)

46. Benrejeb, M, Gasmi, M, Borne, P: New stability conditions for TS fuzzy continuous nonlinear models. Nonlinear Dyn. Syst. Theory 5, 369-379 (2005) 
47. Filali, RL, Hammami, S, Benrejeb, M, Borne, P: On synchronization, anti-synchronization and hybrid synchronization of 3D discrete generalized H12 non map. Nonlinear Dyn. Syst. Theory 12, 81-96 (2012)

48. Benrejeb, M, Borne, P, Laurent, F: Sur une application de la représentation en flèche à l'analyse des processus. RAIRO. Autom. 16, 133-146 (1982)

49. Borne, P, Gentina, JC, Laurent, F: Sur la stabilité des systèmes Échantillonnés non linéaires. Rev. Fr. Autom. Inform. Rech. Opér. 2, 96-105 (1972)

50. Borne, P, Vanheeghe, P, Duflos, E: Automatisation des processus dans l'espace d'état. Editions Technip, Paris (2007)

51. Borne, P: Nonlinear system stability: vector norm approach. In: System and Control Encyclopedia, vol. 5 , pp. 3402-3406 (1987)

52. Kotelyanski, DM: Some properties of matrices with positive elements. Mat. Sb. 31, 497-505 (1952)

53. Robert, F: Recherche d'une M-matrice parmi les minorantes d'un opérateur liéaire. Numer. Math. 9, 188-199 (1966)

54. Gantmacher, FR: Théorie des matrices, Tomes 1 et 2. Dunod, Paris (1966)

10.1186/1687-1847-2014-233

Cite this article as: Kermani and Sakly: On stability analysis of discrete-time uncertain switched nonlinear time-delay systems. Advances in Difference Equations 2014, 2014:233

\section{Submit your manuscript to a SpringerOpen ${ }^{\circ}$ journal and benefit from:}

- Convenient online submission

- Rigorous peer review

- Immediate publication on acceptance

Open access: articles freely available online

- High visibility within the field

- Retaining the copyright to your article 